\title{
Compte rendu de thèse
}

\section{Fabrice Pinteau, Le Tourisme en Croatie. De la création d'une image touristique à son instrumentalisation}

Thèse de doctorat en géographie, université Blaise Pascal (ClermontFerrand II), dirigée par Christian Jamot (soutenue le 30 septembre 2011)

\section{Fabrice Pinteau}

\section{OpenEdition \\ Journals}

Édition électronique

URL : http://journals.openedition.org/tourisme/468

DOI : 10.4000/tourisme.468

ISSN : 2492-7503

Éditeur

Éditions touristiques européennes

\section{Édition imprimée}

Date de publication : 1 décembre 2011

Pagination : 95-98

ISSN : 2109-5671

Référence électronique

Fabrice Pinteau, «Fabrice Pinteau, Le Tourisme en Croatie. De la création d'une image touristique à son instrumentalisation », Mondes du Tourisme [En ligne], 4 | 2011, mis en ligne le 30 septembre 2015 consulté le 22 septembre 2020. URL : http://journals.openedition.org/tourisme/468 ; DOI : https:// doi.org/10.4000/tourisme.468

\section{cc) $(1) \ominus$}

Mondes du tourisme est mis à disposition selon les termes de la licence Creative Commons Attribution - Pas d'Utilisation Commerciale - Pas de Modification 4.0 International. 


\title{
Le tourisme en Croatie
}

\section{De la création d'une image touristique à son instrumentalisation}

\author{
Thèse de doctorat en géographie, université Blaise Pascal (Clermont-Ferrand II), \\ dirigée par Christian Jamot \\ (soutenue le 30 septembre 201 I)
}

FABRICE PINTEAU_[pinteaufabrice@wanadoo.fr]

$\mathbf{L}$ e mémoire de thèse proposé explore un thème jusque-là peu étudié : le tourisme en Croatie. L'objectif de cette recherche est double. Dans un premier temps, il s'agit d'analyser le développement, ou plutôt le renouveau touristique, d'un pays marqué par des années de guerre (de 1991 à 1995) et indépendant depuis une vingtaine d'années seulement. Un autre versant de ce travail s'intéresse à l'image touristique de la Croatie, grâce à une relecture du phénomène touristique qui investit le champ des représentations issues du tourisme.

Il s'agit donc d'examiner à la fois le phénomène touristique mais également l'image de la destination touristique. En cherchant à répondre à la question "Y a-t-il eu une crise liée à la guerre de la fin de la Yougoslavie ou une simple mise entre parenthèses de l'activitépendant le conflit?", l'analyse s'appuie sur une succession d'hypothèses et de déductions qui conduisent de la description du tourisme croate (formes et facteurs) à l'autre interrogation de la thèse, à savoir "Une déformation de l'image touristique promue est-elle lisible?"

Le plan de l'analyse découle donc de ce double questionnement. Avant de décortiquer l'image touristique, et dans un souci d'objectivité, les éléments classiques de l'étude du tourisme sont abordés pour former un socle (ou référentiel) qui permet, par la suite, d'envisager les principales déformations nourries par la promotion de l'image touristique. Pour mener à bien l'analyse de l'image, une connaissance approfondie du tourisme (ou des faits constatés et scientifiquement énoncés) constitue, en effet, une approche préliminaire indispensable. Ce moment incontournable de l'analyse permet une prise de distance, une véritable objectivisation par rapport à l'analyse des représentations. De ce fait, le plan de la thèse s'appuie sur le constat d'une simple mise entre parenthèses du tourisme pendant le conflit. Sont ensuite analysés les différents facteurs qui ont permis ce redémarrage, dont la promotion. L'analyse de l'image est dès lors entamée, au vu des faits précédemment décrits et grâce à l'emprunt d'outils utilisés par d'autres disciplines (sociologie, géopolitique, sciences de la communication, etc.), selon la technique de "l'bybridation" des sciences sociales décrite par Dogan et Pahre ${ }^{(1)}$.

Ainsi, dans la première partie, un cadre théorique a d'abord été déterminé. La problématique $\mathrm{a}$, dès lors, été orientée vers la notion d'“image" touristique, étudiée selon trois déclinaisons : l'image innée (ou fantasmée) d'une Croatie idéalisée avant d'être visitée, l'image vécue ou perçue par les touristes et l'image promue par les professionnels du tourisme (et notamment par l'Office national du tourisme croate, qui contrôle le discours promotionnel et constitue une voix institu- 
tionnelle quasi unique). L'analyse cherche donc ici à comprendre les mécanismes de la construction de l'image de la Croatie liée au tourisme. Se pose donc, inévitablement, la problématique de l'adéquation entre la réalité et les discours qui sont tenus sur elle.

La posture de thèse pose, en effet, le principe que la dialectique entre représentation et réalité - touristique et territoriale - croate n'est pas du seul ressort commercial : d'autres logiques peuvent intervenir, dont celles qui sont du domaine de la construction identitaire, conduisant à penser que l'image promue est, consciemment ou non, instrumentalisée. Ainsi, grâce à l'image diffusée, voire construite, il existe bel et bien une volonté de créer une "identité croate", de la valoriser ou, tout du moins, d'affirmer sa singularité dans la péninsule balkanique. L'image touristique peut, dès lors, être considérée comme un moyen de propagande, voire comme un médium capable de permettre au pays d'être reconnu internationalement. Par analogie avec les théories de la psychanalyse, l'État croate est ici conçu comme un "État adolescent" pour lequel la guerre de la fin de la Yougoslavie est une phase psychanalytiquement enfantine durant laquelle l' "État enfant" a connu un mouvement organisateur du "non", s'affirmant en tant qu'entité indépendante. La période actuelle peut donc être considérée comme une "phase adolescente", au cours de laquelle le pays connaît une période d'angoisse et d'interrogations quant à sa propre identité.

Après ces éléments méthodologiques, la deuxième partie s'intéresse, grâce notamment à l'exploitation de faits statistiques, au phénomène touristique en termes de flux, mais également aux formes de tourisme. Deux éléments clés de l'analyse apparaissent.

- La crise touristique, plus structurelle que conjoncturelle (c'est-à-dire plus liée à la transition du régime socialiste vers une économie de marché qu'à la guerre de la fin de la Yougoslavie), a vite été dépassée grâce au retour rapide de la clientèle, essentiellement européenne. Seul le marché ex-yougoslave se démarque : alors que les Slovènes ont retrouvé, dès 1996, les littoraux croates, le nombre de nuitées réalisées par les habitants des autres "ex-républiques sœurs" est ainsi bien inférieur à celui d'avant-guerre (6,3 millions en 1990 et 1,7 million en 2009). La reconquête du marché ex-yougoslave semble donc très partielle : dans ces conditions, les nationalismes exacerbés ont donc constitué un véritable frein au développement touristique.

- Le tourisme proposé par la Croatie est quasi uniquement balnéaire. Réalisant 96 \% des nuitées, les régions littorales offrent des pratiques touristiques classiques se référant aux "trois S" (sea, sun, sand). Ainsi, l'analyse montre que les autres formes de tourisme (le tourisme urbain, avec essentiellement Dubrovnik et Zagreb, le tourisme de sites naturels, avec le parc de
Plitvice accueillant 928000 visiteurs, le tourisme thermal et celui des sites de guerre) ne constituent que des formes mineures, écrasées par le poids du tourisme balnéaire tant en termes de fréquentation que d'infrastructures d'accueil potentielles. Ce sont donc des éléments annexes du tourisme balnéaire, qui s'enrichit de leur présence. En ce sens, l'analyse ici menée souligne la combinaison entre les différentes formes et pratiques touristiques et décloisonne une classification qui distingue les formes touristiques en les opposant. Elle suggère que le "touriste en Croatie", pouvant avant tout être qualifié de touriste balnéaire, comme le souligne la très forte saisonnalité, peut également diversifier ses pratiques - les sites naturels ou urbains devenant alors de véritables outils marketing, visant à donner un avantage comparatif à la destination.

La troisième partie cherche à expliquer le rapide rattrapage à la suite de la guerre. De multiples facteurs sont évoqués, dont les plus classiques, comme les avantages d'un climat méditerranéen ou encore la forte capacité d'hébergement sur le littoral, la proximité des grands foyers émetteurs en Europe, les $a$ priori favorables concernant la Croatie. Mais, contrairement à l'idée préconçue et souvent relayée par les médias qui fait de la Croatie une “destination nouvelle", l'argumentation proposée insiste sur la place et sur le rôle de l'histoire du développement touristique de la région, en soulignant que le tourisme actuel, 
tant en termes d'infrastructures que de clientèles, est le résultat de nombreux héritages issus de périodes précédentes (fin du XIX ${ }^{e}$ siècle et époque yougoslave). Alors que, en moins d'un siècle, l'espace croate a été contrôlé par cinq États différents (l'Empire d'Autriche-Hongrie, l'Italie républicaine puis fasciste, le royaume de Yougoslavie, la république populaire fédérative de Yougoslavie et enfin la République de Croatie), aucun d'entre eux n'a remis en cause le développement touristique entrepris sous domination austro-hongroise, copiant celui qui avait été amorcé auparavant sur certains littoraux occidentaux.

Dès la fin du XIX ${ }^{e}$ siècle, une véritable tradition touristique se construit en effet avec le développement d'infrastructures ferroviaires et d'hébergement, d'abord sur la riviera d'Opatija puis en Dalmatie centrale, le tourisme étant alors lié, de façon très classique, à l'accessibilité par le train. Mais c'est durant la période titiste que le tourisme croate se développe, notamment sur les littoraux dalmate et istrien, grâce à des programmes d'aménagement et d'investissements massifs de l'État yougoslave ("Adriatique I" entre 1962 et 1969 et "Adriatique II" entre 1969 et 1975). La période socialiste a donc été essentielle pour la Croatie actuelle : c'est elle qui a conforté le principal pôle touristique istrien (qui réalise aujourd'hui 18 millions de nuitées, soit $31 \%$ du total), c'est également elle qui a mis en avant les régions dalmates et notamment les îles.
De plus, les liens forts qui se sont tissés avec certains pays permettent aujourd'hui à la Croatie de bénéficier d'une clientèle diversifiée. Si la période austro-hongroise a en effet favorisé les premiers liens avec les Autrichiens et les Hongrois, l'Istrie italienne n'a fait que renforcer ceux qui existaient déjà entre Rijeka et la bourgeoisie italienne, alors que la période de l'entre-deux-guerres a permis à la côte dalmate de devenir "la mer des Slaves", attirant notamment la clientèle tchèque. Mais c'est grâce à la politique de Tito, se traduisant par une ouverture sur l'Occident, que le littoral croate a su s'affirmer sur le marché de l'Europe occidentale.

En somme, la période austrohongroise tout comme la période yougoslave ont été deux étapes fondatrices qui ont donné à la Croatie actuelle l'image d'une destination balnéaire classique dans les pays d'Europe de l'Ouest. L'analyse montre ainsi une véritable permanence sur le long terme. Il apparaît donc qu'un tourisme "construit", ancien et bénéficiant d'héritages acquiert une capacité à dépasser les crises conjoncturelles. La conclusion est ici qu'il existe un seuil de développement touristique au-delà duquel, malgré les crises géopolitiques, il est impossible de revenir en arrière et de remettre en question totalement le tourisme : est donc ici posé le concept de "résilience touristique".

Une fois ce cadre scientifique énoncé (formes et histoire touristiques), l'analyse s'oriente, dans une quatrième partie, vers un décorticage de "l'image promue", celle que constituent les différentes représentations issues de la promotion officielle, mais également celle qui est véhiculée par les sources d'informations non institutionnelles. L'étude des acteurs (et de l'influence de la voix institutionnelle, par le biais de l'Office national du tourisme croate, créé en 1992), des moyens de communication et de leur méthode est menée en mettant en avant les caractéristiques des différentes images ainsi produites. De nombreuses déformations par rapport à la réalité sont alors distinguées, certaines empruntant, par exemple, des logiques métonymiques où l'exception devient la règle (comme dans le cas des plages de sable pourtant peu fréquentes) ou utilisant des arguments "publicitaires" mensongers (déformation de l'histoire, invention d'un passé, etc.).

Un dévoiement de l'image est alors souligné, la réalité étant tronquée. Dans un premier temps, certaines de ces déformations peuvent trouver une explication du fait d'une logique commerciale classique : mise en valeur du balnéaire par des espaces quasi mythiques proches d'un "paradis perdu" (plages paradisiaques de sable blanc, mais également fantasme du quatrième " $\mathrm{S}$ " $(\operatorname{sex})$, valorisation de pratiques touristiques à la mode (et notamment celles qui sont liées aux sites de "nature vierge"), utilisation du connu (Dubrovnik étant, par exemple, l'élément clé des premières 
campagnes de communication). Mais, les résultats de la recherche montrent que, de façon volontaire ou non, ces dévoiements sont également, dans le cas croate, d'ordre identitaire. La Croatie affirme ainsi, par le biais de son image touristique, des caractéristiques auxquelles elle souhaite être associée, ce qui lui permet de construire son identité territoriale en soulignant ses différences avec ses voisins balkaniques. Ainsi l'affiliation méditerranéenne mais également l'affirmation d'un passé millénaire, ou encore les négligences ou oublis relatifs aux héritages des périodes ottomanes, la diabolisation du titisme et la victimisation liée à la guerre de 19911995 sont autant d'éléments qui témoignent que l'image promue est la traduction d'une certaine quête identitaire liée à une construction nationale. Cette image de la Croatie crée un pouvoir de présence ou pouvoir d'institution : du fait de la promotion, une certaine légitimation a donc lieu. Il s'agit donc d'accroître la notoriété du pays, dans une logique de besoin de reconnaissance et dans le cadre d'une démarche consolidatrice de l'identité telle que les Croates eux-mêmes la conçoivent.

Avant de conclure, un chapitre est consacré aux perspectives de développement et d'évolution du tourisme en Croatie. Ainsi, plusieurs propositions sont faites pour $\mathrm{y}$ favoriser une durabilité du tourisme en liaison avec l'image promue. Parmi celles-ci, on peut retenir la nécessité de mettre en place une véritable politique de protection des espaces littoraux, celle de rechercher de nouvelles clientèles et notamment de reconquérir le marché ex-yougoslave. Certaines propositions essaient également de limiter l'impact de certains "risques" liés au développement du tourisme. D'un point de vue géopolitique, une attention particulière est ainsi portée à l'épineuse question du statut de la propriété, notamment à celui du cadastre et à la spoliation des biens non croates pendant la guerre. Sont également évoqués les courants xénophobes et nationalistes qui critiquent les investissements internationaux et l'appropriation de "la terre croate" par des étrangers. Enfin, les dérives des revendications régionalistes, parfois fortes dans les zones les plus touristiques (Istrie et Dalmatie), sont une source d'inquiétude non négligeable. Du fait des richesses créées, le tourisme peut, en effet, devenir un facteur majeur de clivage de l'unité territoriale croate, alors que les recherches menées dans le cadre de cette thèse ont montré que les organismes institutionnels tentent de se servir du tourisme comme d'un instrument de construction identitaire du territoire. En somme, alors même que le tourisme est utilisé comme un moyen de valorisation identitaire et de construction de l'unité territoriale, cette activité peut elle-même être à l'origine d'une décomposition territoriale, par le biais de laquelle les régions les plus touristiques pourraient vouloir s'affranchir de la capitale zagréboise et des régions de l'intérieur, dont les problématiques politiques sont très différentes des leurs.

Au total, cette thèse, certes centrée sur la Croatie, ouvre de nombreuses pistes de réflexion qui mériteraient d'être prolongées. La première concerne l'efficience de l'image promue d'un strict point de vue commercial, quand l'étude sur la Croatie montre que le facteur primordial est le bouche à oreille : l'impact commercial des politiques de communication touristique n'est-il pas à relativiser ou à chercher dans d'autres cercles que celui de l'attraction de nouvelles clientèles ? La seconde piste concerne les impacts de l'intégration prochaine de la Croatie à l'Europe pour l'identité croate : la valorisation excessive de la "pureté chrétienne" et d'une Croatie Antemurale christianitatis [rempart de la chrétienté] ne va-telle pas à l'encontre du multiculturalisme qui est un des fondements de l'Europe ? Dans la même optique géopolitique, la question du rôle du touriste peut également être soulevée : ce dernier peut-il, en effet, être considéré comme un nouveau genre de diplomate?

Mais c'est surtout le concept de résilience touristique ou de "durabilité touristique" qui méritera de plus amples développements, grâce notamment à des comparaisons avec d'autres destinations ayant connu, ou connaissant actuellement, une crise touristique.

( I) Mattei Dogan et Robert PAHRE, L'Innovation dans les sciences sociales. La marginalité créatrice, Puf, 1991. 